\title{
What is the Risk of Contracting Mycobacterium Tuberculosis form Donated Blood?
}

\author{
*Muringani BN \\ Department of Medical Microbiology, National University of science and technology, Zimbabwe
}

Received: June 15, 2017; Published: June 30, 2017

*Corresponding author: Muringani BN, Department of Medical Microbiology, Faculty of Health Sciences, National University of science and technology, Zimbabwe, Email: beanetsai@hotmail.com

\begin{abstract}
The need for donated blood cannot be underated and it has become very difficult nowadays were many people are living with HIV. It has also become difficult as the adolescent groups which use to donate blood are also living with HIV. This report looked at the people infected with Tuberculosis and the rate at which negative results are given .In this report, 6163 patients were screened for TB using ZN and culture in an African community

Results: The results which were obtained indicate that there is a high chance of getting a blood tainted with TB as from the results of the 6163 samples, $83.52 \%$ were ZN smear negative and had to go for culture which needed more time for the samples to be ruled out as positive or negative TB samples. Of those samples which went for culture, $22.3 \%$ were positive and these were constituted by $48 \%$ of the smear negative samples with $90 \%$ of the culture positives being confirmed to be MTB complex.
\end{abstract}

Conclusion: How safe are the donated bloods if we do not screen for TB infection? Results obtained from this case report leaves a lot to think about in the world of medical sciences.

\section{Introduction}

The need for donated blood cannot be underated and it has become very difficult nowadays were many people are living with HIV. It has also become difficult as the adolescent groups which use to donate blood are also living with HIV. This report looked at the people infected with Tuberculosis and the rate at which negative results are given .In this report, 6163 patients were screened for TB using ZN and culture in an African community. Mycobacterium infection has also increased, with lobal MDR-TB crisis being much more vast than previously estimated $(489,139$ cases of MDR-TB in 2006)requiring a concerted international effort to combat the situation, Coinciding with a huge gape in access to diagnostic and treatment. In the world, less than $5 \%$ have access to proper diagnosis internationally, with only $10 \%$ of MDR -TB estimated to have access to Treatment (MSF Report 20 March 2012).

If weanalyse these statistics, what are the chances of missing a diagnosis and the patient or an individual ending up donating blood during those incentivised blood donation campaigns. Even if we were to screen for TB, rarely do we ask for sputum samples from blood donors, considering the fact that tissue donor recipients have been reported to end up being infected with TB [1,2].

This report looks at the numbers of patients who submitted sputum samples to a referral laboratory in a Southern African laboratory and the results which were obtained of both smear and culture results.

\section{Objective}

To assess the rate of which ZN screening gives negative results from patients who might have disseminated/milliary tuberculosis.

\section{Method}

Sputum samples received in aTB Reference lab were cultured using MGIT Automated instrument using 4\% NaoH decontamination method Positive cultures were confirmed as MTB Complex using ZN smears and TB AgMPT64 Drug susceptibility testing was done on RIF, INH, Streptomycin and Ethambutol using 1\% proportion method

\section{Results}

Culture results and outcomes (Figure 1).

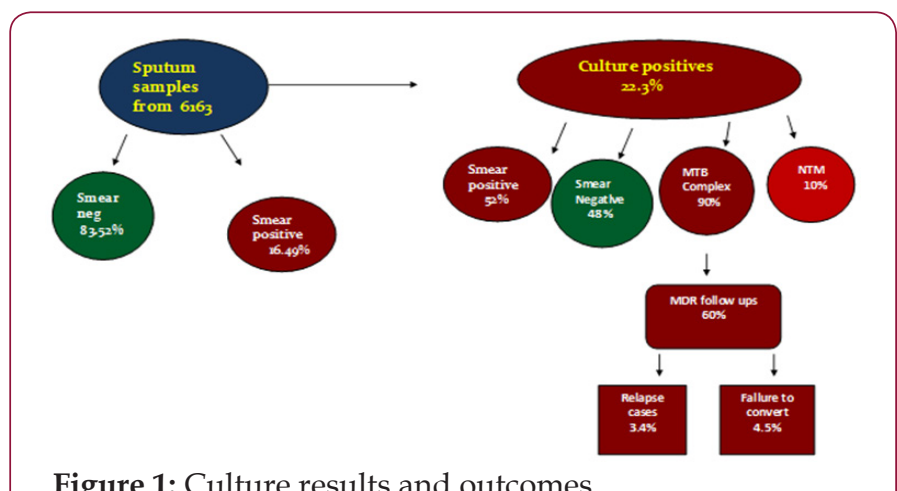




\section{Conclusion}

Over ensuing decades, progress was made in donor-host matching and downward tapering of immunosuppressing regimens, which resulted in improvement in outcomes, but TB remained an uncommon but worrisome complication. In 1983, Lichtenstein and MacGregor [1] reviewed 23 published cases of post-transplantation TB presenting as miliary disease (10 cases [43.5\%]), pulmonary disease (12 cases [52.2\%]) and genitourinary disease (one case [4.3\%]); case mortality was $17.4 \%$ (four of 23 ). This large number of disseminated cases is in contrast with the general population, where $79 \%$ to $84 \%$ of cases are pulmonary [2].

Furthermorethe results which were obtained in our case report indicate that there is a high chance of getting a blood tainted with TB as from the results of the 6163 samples, $83.52 \%$ were ZN smear negative and had to go for culture which needed more time for the samples to be ruled out as positive or negative TB samples. Of those samples which went for culture, $22.3 \%$ were positive and these were constituted by $48 \%$ of the smear negative samples with $90 \%$ of the culture positives being confirmed to be MTB complex.

These results still show that there is need for improved testing methods for TB in the African communities if we are to control spread of TB and also reduce the risk of spreading the disease to unsuspecting Blood recipients as reported by other authors[3-5].

This study did not follow those patients who did not even visit the health centres yet could be harbouring TB due to illness or contact from sick relatives. We only looked at cases which came in for screening and testing yet we still missed the infection in 2470 patients who had turned negative on the ZN screening only to become positive on culture. As other authors have indicated, how safe are the donated bloods if we do not screen for TB infection? $[2,3,6]$ Results obtained from this case report leaves a lot to think about in the world of medical sciences and the need for considering thorough donor screening and also blood screening for other silent infections including MTB.

\section{References}

1. Lichtenstein IH, MacGregor RR (1983) Mycobacterial infections in renal transplant recipients: Report of five cases and review of the literature. Rev Infect Dis 5: 216-226.

2. Centers for Disease Control and Prevention (2003) Reported Tuberculosis in the United States.

3. El-Amine, H EK Bish, D R Bish (2017) Robust post-donation blood screening under limited information on prevalence rates. Forthcoming in Operations Research. Finalist for the 2015 INFORMS PierskallaAward for the Best Paper in Healthcare.

4. Bish DR, EK Bish SR Xie, S Stramer (2014) Going beyond "same-for-all" testing of infectious agents in donated blood. IIE Transactions 46(11): 1147-1168.

5. Bish DR, EK Bish, RS Xie, AD Slonim (2011) Optimal selection of screening assays for infectious agents in donated blood. IIE Transactions on Healthcare Systems Engineering 1(2): 67-90.

6. Xie RS, DR Bish, EK Bish, AD Slonim, SL Stramer (2012) Safety and waste considerations in donated blood screening. European Journal of Operational Research 217(3): 619-632.

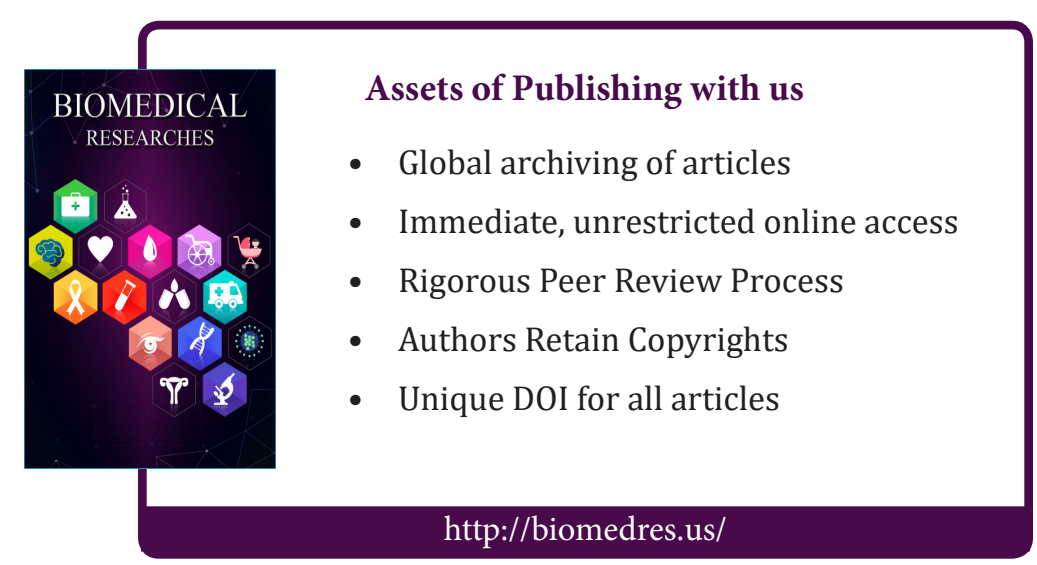

